\title{
O envolvimento do paciente na segurança do cuidado: revisão integrativa
}

\author{
Thaynara de Oliveira Silva ${ }^{1}$, Ana Lúcia Queiroz Bezerra ${ }^{2}$, \\ Thatianny Tanferri de Brito Paranaguá ${ }^{3}$, Cristiane Chagas Teixeira ${ }^{4}$
}

\author{
${ }^{1}$ Discente do curso de graduação em \\ Enfermagem da Faculdade de Enfermagem \\ da Universidade Federal de Goiás. Goiânia, \\ GO, Brasil. E-mail: \\ thaynara_50@hotmail.com. \\ ${ }^{2}$ Enfermeira, Doutora em Enfermagem. \\ Professora Adjunto da Faculdade de \\ Enfermagem da Universidade Federal de \\ Goiás. Goiânia, GO, Brasil. E-mail: \\ analuciaqueiroz@uol.com.br. \\ ${ }^{3}$ Enfermeira, Doutora em Enfermagem. \\ Professora Adjunto da Faculdade de \\ Ciências da Saúde da Universidade de \\ Brasília. Brasília, DF, Brasil. E-mail: \\ ttb.paranagua@gmail.com. \\ ${ }^{4}$ Enfermeira, Mestre em Enfermagem. \\ Goiânia, GO, Brasil. E-mail: $\underline{\text { cc- }}$ \\ teixeira@hotmail.com.
}

Recebido: 17/12/2014.

Aceito: 22/08/2015.

Publicado: 30/06/2016.

Como citar esse artigo:

Silva TO, Bezerra ALQ, Paranaguá TTB, Teixeira CC. O envolvimento do paciente na segurança do cuidado: revisão integrativa. Rev. Eletr. Enf. [Internet]. 2016 [acesso em:____];18:e1173. Disponível em: http://dx.doi.org/10.5216/ree.v18.33340.

\section{RESUMO}

Trata-se de revisão integrativa que objetivou levantar as estratégias adotadas pelas instituições de saúde, que envolvem o paciente no cuidado, como barreira para a prevenção de incidentes. Realizou-se busca nas bases MEDLINE, LILACS, CINAHL e PUBMED, utilizando os descritores "segurança do paciente", "iatrogenia", "erro médico" e "envolvimento". Incluíram-se publicações na íntegra, entre 2003 e março de 2016, nos idiomas inglês, espanhol ou português. Constatouse que a comunicação efetiva e o desenvolvimento da autonomia do paciente são as estratégias mais defendidas. O nível de evidência dos estudos se limitaram a quatro e seis. A avaliação ou descrição de práticas institucionais que envolvem o paciente na sua segurança emerge como lacuna do conhecimento científico. O impacto desta revisão está em constatar a necessidade de estudos randomizados para identificar intervenções efetivas, direcionando as instituições de saúde para a mudança da cultura organizacional, com foco na segurança e no cuidado centrado no paciente.

Descritores: Segurança do Paciente; Doença latrogênica; /prevenção \& controle; Enfermagem.

\section{INTRODUÇÃO}

A temática segurança do paciente tem ganhado enfoque mundial nos dias atuais. A fim de melhorar os processos de trabalho, a partir da redução de erros em saúde e melhoria da segurança, algumas iniciativas têm sido desenvolvidas, dentre as quais se destaca a Aliança Mundial para a Segurança do Paciente ${ }^{(1)}$. Esta aliança foi criada pela Organização Mundial de Saúde (OMS), em 2004, e tem como objetivo principal a 
mobilização de esforços globais para a segurança do cuidado em saúde, direcionando as políticas públicas de saúde no contexto da prevenção de incidentes ${ }^{(1-2)}$.

Entende-se incidente como evento ou circunstância advinda do cuidado, que não possui relação com a doença de base. Os incidentes podem ser classificados como incidentes sem danos, que são aqueles que atingem o paciente, porém não resultam em dano aparente, mas constitui-se um risco; eventos adversos que, obrigatoriamente, resultam em algum tipo de dano ao paciente, seja temporário ou permanente, agravo da situação de saúde, aumento do tempo de internação, exigência de procedimentos adicionais e/ou substituição de tratamento e, até mesmo, o óbito; quase erro que são incidentes que por algum motivo foram interceptados e não atingiram o paciente; e circunstância notificável que é uma situação em que existe potencial para o dano, mas o incidente não acontece ${ }^{(3)}$.

A preocupação mundial com a questão da segurança é decorrente de evidências que comprovam prevalência de $10 \%$ de eventos adversos entre os pacientes que necessitam dos cuidados de saúde ${ }^{(4)}$. No Brasil, estudos apontam prevalência que variam de seis a $18,7 \%$ de eventos adversos ${ }^{(5-8)}$ e incidência de $38,4 \%{ }^{(8)}$. Entretanto, cerca de $66,7 \%$ desses eventos são considerados evitáveis ${ }^{(6)}$.

Estudo retrospectivo realizado na clínica cirúrgica de um hospital universitário evidenciou prevalência de $82 \%$ de incidentes sem dano ${ }^{(7)}$. Dentre os vários tipos de incidentes identificados, destacaram os relacionados ao processo clínico como omissão de cuidado, checagem inadequada e falhas durante procedimentos técnicos. Outros incidentes envolveram os erros de medicação, acidentes com o paciente e, ainda, incidentes relacionados à estrutura organizacional como manutenção de equipamentos, gerenciamento de hemoderivados e de recursos materiais ${ }^{(7)}$.

Constata-se, portanto, que a assistência à saúde pode expor o paciente a várias situações de risco, sendo a hospitalização um fator interveniente para a ocorrência de um evento não esperado ${ }^{(9)}$.

Uma das estratégias que tem sido estimulada para a prevenção desses incidentes é o envolvimento do próprio paciente nas ações de cuidado. Nessa perspectiva a OMS criou o programa Pacientes para a Segurança do Paciente, o qual reúne pacientes, profissionais, gestores e vítimas de danos como parceiros e colaboradores para melhorar a segurança e incentiva que os pacientes sejam colocados no centro dos cuidados e incluídos como parceiros plenos da assistência ${ }^{(10)}$.

Outro programa que visa envolver o paciente no cuidado para prevenir os erros na assistência em saúde é o "Speak Up", criado pela Joint Commission em 2005, que oferece aconselhamentos ao paciente e o estimula a realizar perguntas durante o cuidado, de forma a sanar suas dúvidas e, ainda, participar das decisões ${ }^{(11)}$

Estudo realizado na Dinamarca com pacientes oncológicos constatou que a segurança não era um item que recebia atenção especial dos pacientes e que também não era objeto de comunicação/discussão entre profissionais de saúde ${ }^{(12)}$. Por outro lado, tanto profissionais quanto pacientes afirmaram que o envolvimento do paciente no cuidado é importante por aumentar a segurança da assistência prestada. Dentre os obstáculos para o não envolvimento do paciente, foram destacados o conhecimento limitado sobre os 
aspectos conceituais da segurança do paciente, o desconhecimento de técnicas específicas de envolvimento do paciente na sua segurança e a preocupação com o potencial impacto negativo na relação médicopaciente ${ }^{(12)}$.

Nessa perspectiva, e considerando que o paciente é a última barreira para que o erro aconteça, o presente estudo tem como objetivo levantar as estratégias, utilizadas pelas instituições de saúde, que envolvem o paciente no processo do cuidar, na perspectiva da prevenção dos incidentes, por meio de revisão da literatura.

A relevância do estudo está em identificar, nos estudos mais recentes, evidências da prática clínica, sobre como o garantir o envolvimento do paciente no cuidado, com vistas a melhorar a qualidade e a segurança do paciente durante a assistência à saúde.

\section{METODOLOGIA}

Trata-se de revisão integrativa da literatura que consiste em um método rigoroso para a síntese e análise de conhecimento sobre temática específica, com intuito de facilitar a aplicação dos resultados de estudos significativos na prática clínica ${ }^{(13)}$.

Esse tipo de estudo envolve seis fases: a primeira é a fase de elaboração da pergunta norteadora do estudo; a segunda é a busca ou amostragem da literatura; a terceira constitui na coleta de dados; na quarta se realiza a análise crítica dos estudos incluídos; na quinta se discute os resultados e, por fim, na sexta apresenta-se a síntese do conhecimento ${ }^{(14)}$. Uma das características da revisão integrativa é a investigação por pares, em que dois pesquisadores realizam uma busca independente para posterior discussão e, quando encontram divergências, um terceiro pesquisador é designado.

Especificamente, na quarta fase da revisão integrativa, a literatura selecionada deve ser classificada por nível de evidência, conforme o método utilizado, para auxiliar a avaliação da exatidão e das características dos estudos. O nível 1 contempla evidências da meta-análise de múltiplos estudos clínicos controlados e randomizados; o nível 2 são evidências obtidas em estudos individuais com delineamento experimental; o nível 3 consiste em evidências de estudos quase-experimentais; o nível 4 aponta evidências de estudos descritivos (não-experimentais) ou com abordagem qualitativa; o nível 5 descreve evidências provenientes de relatos de caso ou de experiência e o nível 6 são evidências baseadas em opiniões de especialistas $^{(14)}$.

A questão norteadora adotada para este estudo foi: quais as estratégias utilizadas pelas instituições de saúde que envolvem a participação do paciente na sua segurança durante a assistência à saúde? Para a seleção dos artigos foram realizadas diversas combinações, utilizando os descritores "segurança do paciente", "iatrogenia", "erro médico" e "envolvimento", nas línguas portuguesa e inglesa. As bases de dados consultadas foram MEDLINE, LILACS, CINAHL e PUBMED.

Os critérios de inclusão dos artigos foram: resumo disponível nas bases de dados supracitadas; período de publicação entre janeiro de 2003 e março de 2016, estudos disponíveis na íntegra; publicações nos 
idiomas inglês, espanhol ou português.

A coleta de dados foi realizada por três pesquisadores, de forma independente, finalizada em abril de 2016. As seleções foram confrontadas, a fim de verificar a compatibilidade dos achados e discutir as divergências. Os dados foram sintetizados na forma de quadro para possibilitar análise comparativa entre os estudos e discutidos descritivamente.

\section{RESULTADOS E DISCUSSÃO}

A partir da busca realizada nas bases de dados, foram localizados 21 estudos. Após leitura de títulos e resumos e confronto de dados entre os pesquisadores, foram excluídos cinco estudos que não atenderam aos critérios de inclusão estabelecidos. Foram selecionados dezesseis estudos, sendo localizados seis no MEDLINE, quatro no LILACS, um no CINAHL e cinco na PUBMED. Dois estudos estavam indexados em mais de uma base de dados, totalizando quatorze estudos analisados, os quais estão sumarizados no Quadro 1. 
Quadro 1: Síntese dos estudos sobre o envolvimento do paciente no cuidado como estratégia preventiva de incidentes, organizados por ano de publicação.

\begin{tabular}{|c|c|c|}
\hline Autores. Título / Periódico (Ano) & Objetivo / Tipo de estudo / Nível de evidência & Evidências identificadas nas conclusões dos estudos \\
\hline $\begin{array}{l}\text { Harvard Health Letter. Patient, protect thyself? / } \\
\text { Harvard Health Publications (2006) }\end{array}$ & $\begin{array}{l}\text { Relatar a importância do paciente para a prevenção de } \\
\text { erros. / Opinião de especialistas baseada em literatura } \\
\text { específica. / Evidência } 6 .\end{array}$ & $\begin{array}{l}\text { Envolver o paciente no cuidado reduz a chance de eventos adversos. A } \\
\text { comunicação aberta entre médico-paciente aumenta a segurança. }\end{array}$ \\
\hline $\begin{array}{l}\text { Entwistle VA. Differing perspectives on patient } \\
\text { involvement in patient safety. / Qual Saf Health Care } \\
\qquad(2007)\end{array}$ & $\begin{array}{l}\text { Discutir as vantagens do envolvimento do paciente em } \\
\text { seus cuidados de saúde. / Opinião de especialistas } \\
\text { baseada em literatura específica. / Evidência } 6 .\end{array}$ & $\begin{array}{l}\text { Aponta o envolvimento do paciente como uma questão ética. Dentre as } \\
\text { perspectivas dessa prática estão o respeito ao paciente, melhoria da } \\
\text { comunicação e, ainda, oportuniza o desenvolvimento da autonomia do } \\
\text { paciente e seus familiares. }\end{array}$ \\
\hline $\begin{array}{l}\text { Bartlett G, Blais R, Tamblyn R, Clermont RJ, MacGibbon } \\
\text { B. Impacto dos problemas de comunicação dos } \\
\text { pacientes sobre o risco de eventos adversos evitáveis em } \\
\text { ambientes de cuidados agudos. / CMAJ (2008) }\end{array}$ & $\begin{array}{c}\text { Avaliar a associação entre problema de comunicação e } \\
\text { eventos adversos evitáveis. / Estudo randomizado com } \\
2.355 \text { prontuários de pacientes admitidos em } 20 \\
\text { hospitais gerais de Quebec. / Evidência } 1 .\end{array}$ & $\begin{array}{l}\text { Prevalência de } 2,7 \% \text { de eventos adversos, sendo } 29 \% \text { evitáveis. As admissões } \\
\text { de urgência aumentaram em } 64 \% \text { o risco de evento adverso. Pacientes com } \\
\text { alteração na comunicação foram mais propensos aos eventos adversos ( } 46 \% \\
\text { contra } 20 \%, p=0,05 \text { ). }\end{array}$ \\
\hline $\begin{array}{c}\text { Cernadas JMC. Participación de los pacientes y los } \\
\text { padres en la prevención de los errores en medicina. / } \\
\text { Arch Argent Pediatr. (2009). }\end{array}$ & $\begin{array}{l}\text { Defender a participação do paciente no próprio } \\
\text { cuidado para redução de erros médicos. / Opinião de } \\
\text { especialistas baseada em literatura específica. / } \\
\text { Evidência } 6 .\end{array}$ & $\begin{array}{l}\text { Informar pacientes, pais e/ou acompanhantes sobre o plano de cuidado e } \\
\text { fornecer orientações sobre como auxiliar a equipe de saúde na redução de } \\
\text { erros médicos constitui importante ferramenta para garantir a segurança do } \\
\text { paciente. }\end{array}$ \\
\hline $\begin{array}{l}\text { Bagnulo H, Barbato M, Godino M, Basso J. Evaluación del } \\
\text { riesgo en eventos adversos. / Rev Med Urug (2010) }\end{array}$ & $\begin{array}{l}\text { Abordar áreas temáticas atuais sobre a segurança dos } \\
\text { cuidados. / Opinião de especialistas baseada em } \\
\text { literatura específica. / Evidência } 6 .\end{array}$ & $\begin{array}{l}\text { Destaca a necessidade de pacientes e familiares reconhecerem o evento } \\
\text { adverso; de desenvolver o paciente para o autocuidado; e de orientar } \\
\text { cuidadores com comportamento inadequado ou que refletem cultura } \\
\text { negativa para a segurança. }\end{array}$ \\
\hline $\begin{array}{l}\text { Hall J, Peat M, Birks Y, Golder S, PIPS Group, Entwistle V } \\
\text { et al. Effectiveness of interventions designed to promote } \\
\text { patient involvement to enhance safety: a systematic } \\
\text { review. / Qual Saf Health Care (2010) }\end{array}$ & $\begin{array}{l}\text { Avaliar a eficácia das intervenções de envolvimento do } \\
\text { paciente com o cuidado de saúde. / Revisão } \\
\text { sistemática. / Evidência } 1 .\end{array}$ & $\begin{array}{l}\text { Identificaram } 14 \text { estudos experimentais e quase-experimentais e uma } \\
\text { revisão sistemática. Melhores resultados de incidentes de segurança do } \\
\text { pacientes são encontrados em ambiente que envolvem o paciente no } \\
\text { cuidado. Há evidência limitada sobre a eficácia dessas intervenções. }\end{array}$ \\
\hline $\begin{array}{l}\text { Gallotti RMD, Morinaga CV, Rodrigues MA, Velasco IT, } \\
\text { Martins MA, Tiberio IC. A new method for the } \\
\text { assessment of patient safety competencies during a } \\
\text { medical school clerkship using an objective structured } \\
\text { clinical examination. / Clinics (2011) }\end{array}$ & $\begin{array}{c}\text { Avaliar o desenvolvimento de competências para a } \\
\text { segurança do paciente, por meio do Objective } \\
\text { Structured Clinical Examination (OSCE). / Estudo } \\
\text { descritivo realizado com } 95 \text { estudantes de medicina. / } \\
\text { Evidência } 4 .\end{array}$ & $\begin{array}{l}\text { Menos de } 60 \% \text { dos alunos ofereceram ao paciente um pedido de desculpas } \\
\text { após um erro médico. Aponta o OSCE como ferramenta útil ao } \\
\text { desenvolvimento de competências no relacionamento médico-paciente, o } \\
\text { cuidado centrado no paciente e o reconhecimento do erro, aspectos que } \\
\text { devem ser trabalhados na formação dos profissionais de saúde. }\end{array}$ \\
\hline $\begin{array}{l}\text { Ward K, McEachan RR, Lawton R, Armitage G, Watt I, } \\
\text { Wright J. Patient involvement in patient safety: Protocol } \\
\text { for developing an intervention using patient reports of } \\
\text { organizational safety and patient incident reporting. / } \\
\text { BMC Health Services Research (2011) }\end{array}$ & $\begin{array}{l}\text { Desenvolver ferramenta de registro de incidentes pelo } \\
\text { próprio paciente / Estudo descritivo. / Evidência } 4 .\end{array}$ & $\begin{array}{c}\text { O registro do incidente pelo paciente consiste em meio confiável sobre sua } \\
\text { segurança e traz uma perspectiva diferente quando comparada à do } \\
\text { profissional de saúde, o que promove subsídios para a prática clínica e } \\
\text { aprendizagem organizacional. }\end{array}$ \\
\hline
\end{tabular}

Rev. Eletr. Enf. [Internet]. 2016 [acesso em:___/_];18:e1173. Disponível em: http://dx.doi.org/10.5216/ree.v18.33340. 


\begin{tabular}{|c|c|c|}
\hline $\begin{array}{c}\text { Wegner W, Pedro ENR. Patient safety in care } \\
\text { circumstances: prevention of adverse events in the } \\
\text { hospitalization of children / Rev Latino-Am Enfermagem } \\
\text { (2012) }\end{array}$ & $\begin{array}{l}\text { Analisar a percepção de eventos adversos com } 15 \\
\text { acompanhantes ou cuidadoras e } 23 \text { profissionais da } \\
\text { saúde. / Estudo de caso de abordagem qualitativa, em } \\
\text { unidade de internação pediátrica. / Evidência } 5 .\end{array}$ & $\begin{array}{l}\text { Fiscalização e vigilância pela acompanhante e comunicação efetiva entre } \\
\text { profissional- acompanhante-criança reduzem eventos adversos. A presença } \\
\text { do acompanhante foi requisito para a cultura de segurança na hospitalização } \\
\text { infantil. }\end{array}$ \\
\hline $\begin{array}{c}\text { McDonald KM, Bryce CL, Graber ML. The patient is in: } \\
\text { patient involvement strategies for diagnostic error } \\
\text { mitigation. / BMJ Qual Saf (2013) }\end{array}$ & $\begin{array}{c}\text { Descrever estratégias para o envolvimento do paciente } \\
\text { na redução de erros de diagnóstico. / Opinião de } \\
\text { especialistas baseada em literatura específica. / } \\
\text { Evidência } 6 .\end{array}$ & $\begin{array}{c}\text { Defende o envolvimento do paciente para melhorar a precisão dos } \\
\text { diagnósticos médicos. Direciona os pacientes sobre perguntas que devem } \\
\text { fazer ao profissional que oferece o diagnóstico e encoraja a atitude proativa } \\
\text { do paciente. }\end{array}$ \\
\hline $\begin{array}{l}\text { Hrisos S, Thompson R. Seeing it from both sides: do } \\
\text { approaches to involving patients in improving their } \\
\text { safety risk damaging the trust between patients and } \\
\text { healthcare professionals? An Interview Study. / Plos one } \\
\text { (2013) }\end{array}$ & $\begin{array}{c}\text { Analisar as implicações do envolvimento do paciente } \\
\text { no cuidado. / Estudo qualitativo realizado com } 16 \\
\text { pacientes, quatro familiares e } 39 \text { profissionais de } \\
\text { saúde. / Evidência } 4 .\end{array}$ & $\begin{array}{l}\text { Iniciativas de segurança envolvendo o paciente devem encorajar um trabalho } \\
\text { conjunto entre profissional-paciente, cujo objetivo é aumentar a segurança. } \\
\text { Enfoque colaborativo deve ser ressaltado para evitar tensões negativas na } \\
\text { relação profissional-paciente. }\end{array}$ \\
\hline $\begin{array}{l}\text { Mohsin SS, Sara G, Bryony DFP. Involvement in } \\
\text { medication safety in hospital: an exploratory study. / Int } \\
\text { J Clin Pharm (2014) }\end{array}$ & $\begin{array}{l}\text { Analisar o relato de pacientes sobre o cuidado seguro } \\
\text { com medicamentos e o apoio oferecido pelos } \\
\text { profissionais de saúde para esse envolvimento / Estudo } \\
\text { descritivo realizado com } 100 \text { pacientes e } 104 \\
\text { profissionais de saúde. / Evidência } 4 .\end{array}$ & $\begin{array}{l}\text { Pacientes do sexo feminino e os menores de } 65 \text { anos tiveram nível } \\
\text { significativamente mais elevado de envolvimento. Farmacêuticos e } \\
\text { enfermeiros foram mais propensos a oferecer apoio aos pacientes que fazem } \\
\text { perguntas sobre seus medicamentos. }\end{array}$ \\
\hline $\begin{array}{l}\text { Berger Z, Flickinger TE, Pfoh E, Martinez KA, Dy SM. } \\
\text { Promoting engagement by patients and families to } \\
\text { reduce adverse events in acute care settings: a } \\
\text { systematic review. / BMJ Qual Saf (2014) }\end{array}$ & $\begin{array}{c}\text { Analisar como as intervenções que incentivam a } \\
\text { participação de pacientes e familiares no cuidado têm } \\
\text { sido implementadas. / Revisão sistemática. / Evidência } \\
1 .\end{array}$ & $\begin{array}{l}\text { Identificaram } 18 \text { estudos que evidenciaram a importância de orientar o } \\
\text { paciente para o seu envolvimento no cuidado. Apesar do incentivo para esse } \\
\text { envolvimento na prevenção da infecção hospitalar, de quedas e Pneumonia } \\
\text { associada à Ventilação Mecânica, durante as transições de cuidado e do } \\
\text { checklist cirúrgico, medidas de efetividade são insuficientes. }\end{array}$ \\
\hline $\begin{array}{l}\text { Ridelberg M, Roback K, Nilsen P. Facilitators and barriers } \\
\text { influencing patient safety in Swedish hospitals: a } \\
\text { qualitative study of nurses' perceptions. / BMC Nursing } \\
\text { (2014) }\end{array}$ & $\begin{array}{l}\text { Levantar os fatores facilitadores e dificultadores da } \\
\text { segurança do paciente. / Estudo descritivo, qualitativo, } \\
\text { desenvolvido com } 12 \text { enfermeiros vinculados a } \\
\text { hospitais gerais da Suécia. / Evidência } 4 .\end{array}$ & $\begin{array}{l}\text { Fatores intrínsecos ao paciente podem influenciar a sua segurança. Portanto, } \\
\text { a interação positiva entre profissionais de saúde e pacientes, além do } \\
\text { envolvimento do paciente no cuidado são fatores favoráveis à segurança. }\end{array}$ \\
\hline
\end{tabular}

Rev. Eletr. Enf. [Internet]. 2016 [acesso em:_____];18:e1173. Disponível em: http://dx.doi.org/10.5216/ree.v18.33340. 
O ano de publicação dos estudos variou de 2006 a 2014. Não foram encontradas publicações que tratam, especificamente, sobre o envolvimento do paciente nas questões de segurança durante o período de janeiro de 2015 a março de 2016. Os estudos que abordam a importância de envolver o paciente no cuidado para a redução dos incidentes, se limitaram a métodos com evidência de nível 4 e 6 , que corresponderam a 71,4\% dos achados (10 publicações). Apenas um estudo apresentou nível de evidência 5 e tratou, especificamente, de como acompanhantes/cuidadores e profissionais da saúde reconhecem os eventos adversos nas circunstâncias de cuidado. As melhores evidências, classificadas em nível 1, contemplaram três estudos e evidenciaram a importância da comunicação entre os envolvidos no cuidado e da qualidade das orientações oferecidas ao paciente/acompanhantes para a manutenção da segurança.

A presente revisão integrativa identificou como lacuna do conhecimento científico, estudos que avaliam ou que descrevem a prática de programas institucionais que envolvem o paciente no cuidado nas instituições de saúde, com foco na redução de incidentes e melhoria da segurança.

A análise dos resultados permitiu a distribuição desses em duas categorias: "Estratégias de envolvimento do paciente no cuidado seguro" e "Educação do profissional de saúde para o envolvimento do paciente no cuidado". A discussão apresentada versa sobre vários aspectos que convergem para a comunicação, que emergiu como uma ferramenta essencial para a gestão do cuidado seguro.

O impacto destes resultados está na evidência de que muito se deve avançar nas ações que envolvem o paciente como eixo central do cuidado, considerando-o como a última barreira para evitar a ocorrência de um incidente, prática ainda incipiente na rotina dos serviços de saúde. Evidenciou-se que o cenário de estudo dos artigos foi o hospitalar, havendo necessidade de ampliar as discussões sobre o envolvimento do paciente na segurança da assistência à saúde em outros cenários de prática, incluindo a atenção primária à saúde e em instituições com serviços especializados.

Estratégias de envolvimento do paciente para o cuidado seguro

Foram encontradas como estratégias de envolvimento do paciente no cuidado seguro a comunicação aberta/efetiva entre pacientes, acompanhantes e profissionais de saúde; o desenvolvimento da autonomia do paciente para o autocuidado; o envolvimento do paciente no cuidado; a disponibilidade de manuais e/ou cartilhas explicativas sobre o processo patológico do paciente; e a capacitação dos pacientes para a percepção dos riscos.

A interação entre profissionais de saúde e paciente pode facilitar ou dificultar a manutenção da segurança ${ }^{(15)}$. Ambientes em que o paciente não recebe informações sobre o cuidado, onde a interação profissional-paciente é considerada baixa, são considerados ambientes inseguros ${ }^{(15)}$. Por outro lado, uma interação positiva pode favorecer a segurança.

Nessa perspectiva, a comunicação é uma ferramenta de extrema importância para a efetiva relação entre profissional e paciente e que contribui, diretamente, para a prevenção de incidentes ${ }^{(16-17)}$, além de ser apontada como uma questão ética no contexto da assistência à saúde ${ }^{(18)}$. 
Para que uma comunicação efetiva aconteça é necessário utilizar linguagem clara e objetiva, evitar terminologias técnicas, fornecer informações completas e precisas aos pacientes e permitir/oportunizar o esclarecimento de dúvidas ${ }^{(17)}$. Além disso, deve ser estabelecida uma comunicação cada vez mais aberta ${ }^{(16)}$, de modo que esta relação seja simétrica e minimize possíveis relações de poder.

No contexto das internações pediátricas, especialmente, o estabelecimento de uma comunicação aberta e efetiva entre profissionais de saúde e acompanhantes tem sido incentivado ${ }^{(17,19-21)}$, o que é influenciado pela complexidade dos cuidados e fragilidade do paciente.

Dentre as estratégias de envolvimento do paciente para a redução de incidentes destacam-se a orientação de pacientes, pais e/ou acompanhantes sobre o plano de cuidados, o oferecimento de informações sobre como podem auxiliar a equipe de saúde na redução de erros médicos e, ainda, o incentivo de sua participação na fiscalização e vigilância dos cuidados ${ }^{(17)}$. Entretanto, devem ser estratégias bem trabalhadas pela instituição de saúde, de forma a evitar possíveis tensões e sentimentos de desconfiança na relação paciente-profissional ${ }^{(22)}$. Garantir um diálogo aberto com os pacientes para que eles saibam quem contatar quando algo dá errado favorece, positivamente, a segurança, pois podem auxiliar na previsão de situações de risco iminente ${ }^{(15)}$.

Constata-se que pacientes com problemas de comunicação são mais propensos aos eventos adversos quando comparados aos pacientes sem esses problemas, numa proporção de $46 \%$ contra $20 \%$, o que comprova a importância da comunicação efetiva para a melhoria da segurança do paciente ${ }^{(20)}$.

$\mathrm{Na}$ tentativa de sanar possíveis problemas na relação profissional-paciente, muitas vezes existentes em condições fisiológicas normais, o reconhecimento precoce de problemas na comunicação do paciente, por meio de avaliação profissional, no primeiro contato, pode auxiliar a adoção de medidas necessárias que minimizem as interferências inter-relacionais ${ }^{(20)}$.

O empoderamento do paciente no cuidado também constitui estratégia de redução de incidentes e está diretamente relacionada à comunicação entre profissionais e pacientes ${ }^{(17)}$. Pacientes empoderados interagem de forma eficaz e contribuem para a obtenção de melhores resultados ${ }^{(23)}$. Para isso, orienta-se conscientizar os pacientes sobre a situação de saúde, sobre as questões de segurança e, ainda, considerar que muitos pacientes podem estar hospitalizados pela primeira vez ${ }^{(15)}$. Assim, informações básicas sobre o processo de trabalho em saúde podem favorecer a segurança do paciente.

É importante que o paciente conheça e questione sobre os procedimentos aos quais será submetido. Em contra partida, o profissional deve informar o paciente sobre questões relacionadas a seu estado de saúde, estabelecer uma relação de confiança, e propiciar a troca de informações ${ }^{(21,24)}$. Cabe ao profissional, portanto, no contexto da segurança, estimular os pacientes a desenvolverem conhecimento e habilidades que os auxiliem nas decisões acerca de sua saúde.

A disponibilização de cartilhas sobre diferentes patologias e tratamentos é uma estratégia que favorece o entendimento do paciente a cerca do seu problema de saúde e o capacita para a discussão e esclarecimento de determinadas dúvidas com os profissionais ${ }^{(24)}$. Avaliar o entendimento do paciente sobre 
as orientações/informações fornecidas aumenta a segurança do paciente ${ }^{(15)}$, portanto deve ser uma prática sistemática.

O envolvimento do paciente no autocuidado está intimamente ligado à relação estabelecida com o profissional de saúde. A base para esta relação deve ser a confiança, o respeito ao questionamento do paciente e a escuta de suas dúvidas, estabelecendo o vínculo e desenvolvendo sua autonomia ${ }^{(23)}$.

Apesar do incentivo ao envolvimento do paciente no cuidado para aumentar a sua segurança, pouco se observa sua aplicabilidade pelas instituições de saúde ${ }^{(25)}$. Estudo realizado através de vasta busca na literatura apontou que é limitada a evidência de intervenções eficazes dessa prática ${ }^{(26)}$, o que pode estar relacionado à baixa adesão dos profissionais de saúde. Dessa forma, intervenções devem ser analisadas a fim de ampliar as perspectivas do envolvimento real do paciente no cuidado, de forma que este seja o mais próximo possível do envolvimento considerado desejável ${ }^{(27)}$.

Destaca-se que o desenvolvimento da autonomia de pacientes, pais e/ou acompanhantes para o cuidado refletem na mudança da cultura organizacional, especialmente, na abordagem do erro em saúde ${ }^{(17,19-21)}$. Encorajar um papel mais proativo do paciente no seu próprio cuidado e segurança deve ser uma ação institucional, o que torna mais aceitável e praticável tanto para pacientes, quanto para os profissionais $^{(22)}$.

\section{Educação do profissional de saúde para o envolvimento do paciente no cuidado}

A prática de envolver o paciente no cuidado, no contexto das instituições de saúde, parece não ter a mesma proporção que os incentivos da comunidade científica. Isso revela a necessidade de investir na formação dos profissionais de saúde, no intuito de promover mudanças atitudinais e, como resultado, conseguir melhores resultados assistenciais.

A notificação do erro pelo profissional é considerada ferramenta importante para as instituições de saúde, visto que direcionam as ações gerenciais e assistenciais para cada indicador e/ou falha identificada ${ }^{(17)}$. A roda de conversa é uma técnica que pode estimular os profissionais de saúde a discutirem os incidentes e oferece oportunidades para assunção de um erro. Além disso, proporciona o estudo coletivo para o reconhecimento das falhas e influencia no desenvolvimento da autonomia profissional para agir de forma responsável e prudente, além de favorecer a tomada de decisão mais assertiva ${ }^{(17)}$. Reconhecer o erro é, portanto, etapa inicial para a promoção de mudanças no processo de trabalho.

Nesse mesmo sentido, o envolvimento do paciente na abordagem do erro em saúde tem sido destacado pelo fato de o paciente apresentar uma visão diferente e, portanto, possibilitar a percepção de outras causas e/ou fatores que por ventura não foram identificados pelos profissionais de saúde. Ressaltase a importância do treinamento desses profissionais para que saibam como relatar ao paciente a ocorrência de um incidente. A forma de abordar o incidente influencia na decisão do paciente em levar ou não o ocorrido aos trâmites judiciais ${ }^{(24)}$, lembrando que o foco principal dessa prática é a mitigação do erro em saúde para ampliar a capacidade da instituição em agir preventivamente. 
O uso da técnica OSCE (Objective Structured Clinical Examination) como instrumento de avaliação do desempenho dos alunos de graduação em medicina verificou que a avaliação das habilidades dos alunos no domínio "erro médico" obteve nota significativamente menor do que as habilidades nos domínios "relação entre médico-paciente" e "questões de humanização". Verificou-se, ainda, que dentre os alunos que abordaram o erro médico com os pacientes, menos de $60 \%$ ofereceram ao paciente um pedido de desculpas ${ }^{(19)}$.

Essa realidade evidencia o desafio das instituições de ensino para a formação de profissionais proativos, com habilidades de relacionamento interpessoal e para a adoção práticas e condutas que convergem para a cultura de segurança. Mais ainda, reflete no desafio de incorporar na postura profissional a importância da assistência centrada no paciente, transferindo para este, uma autonomia para agir em prol da qualidade e segurança da sua própria assistência, sem parecer um confronto ${ }^{(18)}$. Essa "nova" perspectiva para o cuidado exige a habilidade de estabelecer um diálogo que permita investigar de forma acurada o contexto socioeconômico e cultural que o paciente está inserido, respeitando as diferenças e direcionandoo para uma assistência integral, em substituição da atenção curativista ${ }^{(23)}$.

A maioria dos estudos enfatiza a ocorrência dos eventos adversos, ou seja, quando o dano já aconteceu. Disseminar os conceitos de near misses e circunstâncias notificáveis torna-se necessário por acreditar que a reflexão de ações que não causaram dano ao paciente reduz o medo da punição e ampliam as possibilidades de envolver o paciente na tomada de decisões preventivas, de modo a romper barreiras no processo do cuidar e aperfeiçoar a relação profissional-paciente, preservando todas as características da comunicação efetiva, ora já discutidas.

Frente a isso, afirma-se, seguramente, que o meio eficaz de gerenciar a segurança do paciente continua sendo as intervenções colaborativas, envolvendo pacientes, profissionais de saúde e pesquisadores $^{(28)}$.

Opinião de especialistas incentiva o envolvimento do paciente, inclusive, para aumentar a precisão dos diagnósticos médicos, o que exige a autonomia do paciente para levantar questionamentos/considerações sobre a informação recebida pelo profissional de saúde ${ }^{(29)}$. Entretanto, apenas diante de uma gestão cuidadosa, com foco no desenvolvimento de competências para a segurança, é que a prática de envolver o paciente no cuidado exercerá sua função plena no trabalho de melhoria da qualidade dos serviços de saúde.

\section{CONCLUSÃO}

As seguintes estratégias de envolvimento do paciente no cuidado têm sido defendidas: adoção de comunicação aberta e efetiva entre profissionais-acompanhantes-pacientes; fiscalização e vigilância dos cuidados pela acompanhante; permanente conferência e revisão das intervenções profissionais pelo paciente; reconhecimento dos eventos adversos pelos pacientes e familiares; fornecer informações aos pacientes, pais e/ou acompanhantes sobre o plano de cuidado; fornecer orientações aos pacientes sobre como auxiliar a equipe de saúde na redução de erros médicos; informar pacientes e familiares sobre a 
ocorrência de eventos adversos; e promover o compromisso do paciente com o autocuidado.

Não foram encontrados estudos que avaliam a eficácia dessas estratégias para a redução de incidentes, constituindo-se essas, lacunas do conhecimento. Também não foram identificados estudos que descrevem a prática de envolvimento do paciente no cuidado como um programa institucional. Diante dessas evidências, recomenda-se que novas pesquisas sejam desenvolvidas, focadas na importância do envolvimento do paciente no próprio cuidado, na utilização, na contribuição e na eficácia dessa prática para a redução de incidentes e visando o aprimoramento do processo de trabalho nos serviços de saúde.

As evidências apontam para a pouca disseminação da cultura de envolvimento do paciente, na perspectiva da segurança do cuidado. Aponta, também, para uma temática incipiente no contexto de avaliação de estratégias que reduzam os incidentes. Os resultados desta revisão integrativa apresentam limitações pelo fato de grande parte dos estudos constituírem níveis de evidência 6, o que levanta um alerta para a comunidade científica sobre a necessidade de estimular pesquisas com métodos mais robustos, com foco na instrumentalização dos profissionais de saúde para o cuidado centrado no paciente e sua família, prática altamente recomendada nos dias atuais.

\section{REFERÊNCIAS}

1. World Health Organization. Word alliance for patient safety. Forward program 2006-2007 [Internet]. Genebra (SW); 2007 [cited 2014 jan 20]. Available from: http://www.who.int/patientysafety.

2. Dias MAE, Martins M, Navarro N. Rastreamento de resultados adversos nas internações do Sistema Único de Saúde. Rev Saúde Pública. 2012;46(4):719-29.

3. World Health Organization. World alliance for patient safety. Taxonomy. The conceptual framework for the international classification for patient safety [Internet]. Genebra (SW); 2009 [cited 2013 dez 19]. Available from: http://www.who.int/patientsafety/taxonomy/icps_full_report.pdf.

4. World Health Organization. World alliance for patient safety. Guideline safe surgery [Internet]. 1st ed. Genebra (SW); 2008 [cited 2013 mar 20]. Available from:

http://www.who.int/patientsafety/safesurgery/knowledge_base/SSSL_Brochure_finalJun08.pdf

5. Capucho HC, Arnas ER, Cassiani SHBD. Segurança do paciente: comparação entre notificações voluntárias manuscritas e informatizadas sobre incidentes em saúde. Rev Gaúcha Enferm. 2013;34(1):164-72.

6. Mendes W, Martins M, Rozenfeld S, Travassos C. The assessment of adverse events in hospitals in Brazil. Int J Qual Health Care. 2009;21(4):279-84.

7. Paranaguá TTB, Bezerra ALQ, Silva AEBC, Azevedo Filho FM de. Prevalence of no harm incidents and adverse events in a surgical clinic. Acta Paul Enferm. 2013;26(3):256-62.

8. Mendes W. Mendes W, Travassos C, Martins M, Noronha JC de Revisão dos estudos de avaliação da ocorrência de eventos adversos em hospitais. Rev Bras Epidemiol. 2005;8(4):393-06.

9. Organização Pan-Americana de Saúde. Aliança mundial para a segurança do paciente: cirurgias seguras salvam vidas. Rio de Janeiro: Organização Pan-Americana de Saúde; 2009.

10. World Health Organization. Patients for Patient Safety: forward program [Internet]. Genebra (SW); 2004 [cited 2014 jan 05]. Available from: http://www.who.int/patientsafety/patients_for_patient/en/.

11. Joint Commission on the Accreditation of Healthcare Organizations. "Speak Up" [Internet]. USA; 2005 [cited 2014 jan 15]. Available from: http://www.jointcommission.org/assets/1/18/speakup_amb.pdf.

12. Martin HM, Navne LE, Lipczak H. Involvement of patients with cancer in patient safety: a qualitative study of current practices, potentials and barriers. BMJ Qual Saf. 2013;22(10):836-42.

13. Coscrato G, Pina JC, Mello DF. Use of recreational activities in health education: Integrative review of literature. Acta Paul Enferm. 2010;23(2):257-63.

14. Souza MT, Silva MD, Carvalho R. Revisão integrativa: o que é e como fazer. Einstein. 2010;8(1):102-6.

Rev. Eletr. Enf. [Internet]. 2016 [acesso em:___/__;18:e1173. Disponível em: http://dx.doi.org/10.5216/ree.v18.33340. 
15. Ridelberg M, Roback K, Nilsen P. Facilitadores e barreiras influenciar a segurança do paciente em hospitais suecos: um estudo qualitativo de percepção das enfermeiras. BMC Nurs. 2014; 13: 23.

16. Harvard Health Letter. Patient, protect thyself? Harvard Content. 2006.

17. Wegner W, Pedro ENR. Patient safety in care circumstances: prevention of adverse events in the hospitalization of children. Rev Latino-Am Enfermagem. 2012;20(3):427-34.

18. Entwistle VA. Differing perspectives on patient involvement in patient safety. Qual Saf Health Care. 2007;16(2):823.

19. Gallotti RMD, Morinaga CV, Rodrigues MA, Velasco IT, Martins MA, Tiberio IC. A new method for the assessment of patient safety competencies during a medical school clerkship using an objective structured clinical examination. Clinics. 2011;66(7):1209-15.

20. Bartlett G, Blais R, Tamblyn R, Clermont RJ, MacGibbon B. Impacto dos problemas de comunicação dos pacientes sobre o risco de eventos adversos evitáveis em ambientes de cuidados agudos. CMAJ. 2008;178(12):1555-62.

21. Cernadas JMC. Participación de los pacientes y los padres en la prevención de los errores en medicina. Arch Argent Pediatr. 2009;107(2):97-8.

22. Hrisos S, Thompson R. Seeing it from both sides: do approaches to involving patients in improving their safety risk damaging the trust between patients and healthcare professionals? An Interview Study. Plos one. 2013;8(11).

23. Taddeo PS, Gomes KWL, Caprara A, Gomes AMA, Oliveira GC, Moreira TMM. Acesso, prática educativa e empoderamento de pacientes com doenças crônicas. Ciênc saúde coletiva. 2012;17(11):2923-30.

24. Bagnulo H, Barbato M, Godino M, Basso J. Evaluación del riesgo en eventos adversos. Rev Med Urug. 2010;26(1):55-7.

25. Berger Z, Flickinger TE, Pfoh E, Martinez KA, Dy SM. Promoting engagement by patients and families to reduce adverse events in acute care settings: a systematic review. BMJ Qual Saf [Internet]. 2013 [acesso em: 30 jun. 2016]. Disponível em: https://doi.org/10.1136/bmjqs-2012-001769.

26. Hall J, Peat M, Birks Y, Golder S, PIPS Group, Entwistle V et al. Effectiveness of interventions designed to promote patient involvement to enhance safety: a systematic review. Qual Saf Health Care. 2010;19(5).

27. Mohsin SS, Sara G, Bryony DFP. Involvement in medication safety in hospital: an exploratory study. Int J Clin Pharm. 2014;36(3):657-6.

28. Ward JK, McEachan RR, Lawton R, Armitage G, Watt I, Wright J. Patient involvement in patient safety: Protocol for developing an intervention using patient reports of organisational safety and patient incident reporting. BMC Health Services Research. 2011;11:130.

29. McDonald KM, Bryce CL, Graber ML. The patient is in: patient involvement strategies for diagnostic error mitigation. BMJ Qual Saf. 2013;0:1-7. 